\title{
Research on Chinese - English Advertising Translation Based on Functional Theory
}

\section{Rui Han}

\author{
Heilongjiang University of Technology, Jixi, Heilongjiang, 158100
}

\author{
Keywords: Advertising Translation, Functional Theory, Chinese-English Translation
}

\begin{abstract}
The traditional studies of advertisement translation mainly focus on literature translation, which investigate the style of advertisements through analysis of the lexical and syntactic features and probe into the translation approaches with contrast between the English and Chinese styles of advertisements. Unfortunately, it is largely neglected to study the translation of product advertisements in relation to the theory of functional equivalence. Based on researches, this thesis tries to link the wording of advertisements with its actual context and meaning, and attempts to examine how functional equivalence is reflected in the translation of commercial advertisements in the hope of deepening the understanding of commercial advertisement translation and the theory of functional equivalence.
\end{abstract}

\section{Introduction}

It originates in the 1950s with the Russian-born American structuralist Roman Jakobson who is an early pioneer in applying linguistics into the study of translation. Jakobson follows the relation set out by Saussure between the signifier (the spoken and written signal) and the signified (the concept signified), and points out (Jakobson 1959/2000: 114) that "there is ordinarily no full equivalence between code-units" since they belong to two different sign systems (languages) which partition reality differently. "The translator recodes and transmits a message received from another source. Thus translation involves two equivalent messages in two different codes. "Equivalence in difference is the cardinal problem of language and the pivotal concern of linguistics" (Jakobson1959/2000: 120). Chomsky’s generative-transformational model analyzes sentences into a series of related level governed by rules. He put forward the concept of kernel sentences. Nida incorporates key features of Chomsky's model into his science of translation. He develops a more systematic approach of functional equivalence by borrowing theoretical concepts and terminology both from semantics and pragmatics and from Chomsky's work on syntactic structure. On the other hand, the globalization of economies and trade intensification lead companies to communicate with consumers of different languages and cultures. Within the framework of international marketing strategies, advertising plays a key role. It has to resolve a dilemma which can be summarized in the following question: How can we sell a standardized product to local and different consumers? Therefore, good translations of both Chinese and English advertisements are urgently needed [1].

\section{The Role of Classification of Text Types}

Having seen the source text "dethroned" and regarded as a mere "offer of information" or the translator's "raw material" (Vermeer 1987b: 541), one might be surprised to find that one of the specific theories in Reiss and Vermeer's book is Katharina Reiss's theory of text types. This has to be appreciated in connection with Reiss's concept of a specific translation type referred to as communicative translation, which is associated with a certain notion of equivalence. The theory of text type builds on the concept of equivalence but views the text, rather than the word or sentence, as the level at which communication is achieved and at which equivalence must be sought (Reiss 1977/89: 113- 4). Her functional approach aims at systematizing the assessment of translations. According to Reiss, text typologies help the translator specify the appropriate hierarchy equivalence levels needed for a particular translation skopos. Like several other German linguists and translation 
scholars, Reiss (1989: 105) distinguishes between two forms of text categorization, which are located on different levels of abstraction: on the one hand, text types are classified according $t$ the dominant communicative function (basically informative, expressive or operative); on the other hand, text genres or varieties are classified according to linguistic characteristics or conventions like those of reference books, lectures, satires or advertisements) [2].

Reiss's text typology, first published in 1968-69, is based on the "organ model" of language functions proposed by the German psychologist Karl Buhler in 1934. The main characteristics of each type are informative, expressive and operative. In this way, a reference book is the most fully informative text type; the poem is a highly expressive, form-focused type; and an advertisement is the clearest operative text type which is intended to persuade someone to buy or do something). Between these poles are positioned a host of hybrid of types. Thus, a biography might be somewhere between the informative and expressive types, since it provides information about the subject while also partly performing the expressive function of a piece of literature. Despite the existence of such hybrid types, Reiss (1977/89: 109) states that "the transmission of the predominant function of the source text is the determining factor by which the target text is judged". She suggests "specific translation methods according to text type".

\section{Functional Equivalence at the Level of the Discourse}

According to Reiss, the translator has to take account of the way linguistic signs are used by communicative agents to achieve textual equivalence. Different functions of texts usually mean different forms of equivalence required for an adequate translation. For example, equivalence at word rank does not imply textual equivalence while equivalence at text rank does not automatically lead to lexical or syntactic equivalence. As Reiss suggests, translating operative texts into operative texts ought to be guided by the main purpose of evoking the same reaction in the audience and this might damage the equivalence at the word or syntax rank. Then let's look into a number of advertisements and their translations to see whether or how functional equivalence manifests itself at the level of discourse [3].

The Chinese translation can be considered a functional equivalent of the English original because it is apt to fulfill the same communicative function. First of all, the text "It starts with twin blades, individually and independently mounted on highly responsive springs. So they continuously sense and automatically adjust to the individual curves and unique needs of your face. The new blades are $50 \%$ narrower than any others - allowing water to flow freely around and through them, for effortless cleansing and rinsing. All these Sensor technologies combine to give your individual face a personalized shave-the closest, smoothest, safest, most comfortable." is slightly reconstructed and adapted. Its translation is not completely an equivalent of the source text at the word rank. The translator has offered same adequate information in a different form. In this way, it has achieved textual equivalent and the reader should be able to appreciate the information in the same way as the original audience do. Secondly, the last sentence "The best shave a man can get.

In the Chinese translation, the sentence "A true American classic, Maxwell House is a special blend of high-quality coffee beans expertly roasted to deliver a rich, full-flavored, consistently great-tasting coffee." This translation does not follow the syntactical structure of the original sentence, yet it has conveyed the information of the original and thus is apt to fulfill the communicative function [4]. At the same time, it is not hard to see that the phrase "A true American classic" is not rendered in the target text. Although it might fail to achieve an equivalence at word rank, it does not result in an inadequacy at textual level. It does not make a big difference whether the audience knows that this kind of coffee is an American classic. An advertisement is assumed to brag about the product, so a word like classic will not have much influence on enhancing the attraction of the product or the advertisement. Moreover, since the target market or culture is the Chinese one, "A true American classic" might not be able to touch people's heart as deeply as it does to the source audience. What people in the target culture care about is the product itself. In this way, the absence of "A true American classic" will not affect the communicative function.

First of all, I should admit that the first translation does achieve a kind a equivalence, although 
not functional equivalence. It is obvious that the wording and structure of the first translation is much closer to the source text than those of the second translation. So there is no denying the fact that the first translation has successfully achieved equivalence at word and syntax level, and it is expressive and elegant to some degree. However, as an advertisement, it is quite out of place because of not being concise and powerful enough to fulfill the function of being operative, which should be a major weakness for an advertisement. Due to the differences between the languages involved, the original text is simple and succinct while the translated text reads like an informative introduction of a jeans product. Such a translation does not strike an effective communication with potential customers since people might feel the advertisement is too long and too redundant. Also people might feel it is not able to catch the core message easily and they might even not have much patience to stay through the long delivery of information. Therefore, as far as functional equivalence is concerned, this translation is far from satisfactory as an advertisement translation. In other words, the first translation does not achieve the effects of functional equivalence [5].

\section{Functional Equivalence at the Level of the Receiver}

Receiver is also one of the extralinguistic criteria put forward by Reiss. The target audience is a major factor in determining the translation strategies and the level of language to be employed. According to Reiss, the target text of an operative text should produce the desired response in the target receive. In circumstances when the intended audience is generally of a lower educational level than the audience of the original text. In such cases, a translator must not only do a better job in reflecting the stylistic potentials of the target language but must make adjustments for the differences in educational levels. This usually means simplification of vocabulary and grammar, as well as certain important shifts in dealing with rhetorical features. Reiss observes that "not every language group ... will have the same response to the same kind of advertisement”

Consider that the source text in question is an American cook book for children aged about ten and the target texts will be produced in various languages for sale abroad. The function of the target text is to function in the target culture as an independent message-transmitting text, with the target text receivers using it to learn how to cook. There are several difficulties that the translator will come across. If a utensil mentioned in the book does not exist in the target country, the translation has to suggest a different utensil to finish the cooking job. The presupposition in the source text is that the child will have seen these dishes and understand what the final product is to look like. In target cultures where these dishes are unknown, the children and adults may be unsure whether the recipe is turning out correctly. Changing the names of some of the recipes (for example to Chinese vegetables, exotic rice and hot fruit dessert) may make them more accessible to the target text receivers, although not necessarily easy to cook. In addition, since the audience of the discourse is children, the words in the source text are consequently simplified and interpersonal, using bubbling very gently instead of the word simmer. The translator should pay attention to the choice of words in the same manner [6].

On the other hand, receivers of different cultures are likely to have different interpretations of the same words or phrases, because source receivers and target receivers have different cultural values. Differences of cultural values are important factors in understanding a series of related terms, for example, nigger, negro, colored, black and Afro-American representing in each instance a desire to avoid or to employ expressions that are culturally insulting. Plus, cultural values may influence the associative meanings of a term. In Western Europe, terms for a pig normally have negative associative meanings since pig do not rate high as pets, but in some countries, pigs are important status symbols and the corresponding names for such animals have very positive associative meanings [7]. In American English, the terms such as apple pie, ice cream, stars and stripes, and mother have strong positive associative meanings while cancer and Mafia usually have very unfavorable associative meanings. 


\section{Conclusions}

Although the functional equivalence theory is a relatively comprehensive framework for understanding a text within its context, nevertheless, it cannot possibly embrace everything in the study of advertisement translation. So many newer insights are waiting to be generated and it is hoped that this special issue will encourage further analysis of this genre by scholars working from different areas of translation studies.

\section{References}

[1] Tan Weiguo translation of English and Chinese ad rhetoric [J]. China Translation .2003 (02): $11-18$

[2] Duan Shengfeng, Li Gang. Vocabulary ideas of English and Chinese advertising [J]. China Science and Technology Translation. 2001 (02): 19-27

[3] Hao Qinhai. Cross-cultural Pragmatic Failure in Advertising Language - An Analysis of Advertising Language in Chinese-English Translation [J]. Foreign Language Education. 2000 (03): 54-56

[4] $\mathrm{Hu}$ Kaibao, Chen Zaiquan. Aesthetic characteristics of trade names and the translation of English trade names [J]. Chinese Translator. 2000 (05): 6-9

[5] Yan Xiaoya. On commercial advertising translation [J]. Zhanjiang Normal College. 2000 (03): 98-99

[6] Hao Qinhai. Cultural Connotation in Advertising Language - Cultural Contrast between Chinese and English Advertising Language [J]. Shandong Foreign Language Teaching. 2000 (02): 127-129

[7] Yang Quanhong. Translator, try to explore the international advertising unconventional translation method [J]. China Science and Technology Translation. 2000 (01): 192-201 